\title{
Spread of multidrug resistance among Ureaplasma serovars, Tunisia
}

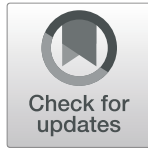

Safa Boujemaa', Béhija Mlik', Amina Ben Allaya ${ }^{1}$, Helmi Mardassi ${ }^{2}$ and Boutheina Ben Abdelmoumen Mardassi ${ }^{*}$

\begin{abstract}
Background: Ureaplasma spp. have been implicated in a variety of clinical conditions and certain serovars are likely to be disease-associated. Hence, the ascending trend of Ureaplasma spp. resistance to antimicrobials should deserve more attention. Here we assessed the extent of antimicrobial resistance of Ureaplasma serovars in Tunisia, and investigated the underlying molecular basis.

Methods: This study included 101 molecularly typed Ureaplasma spp. clinical strains isolated over a 12-year time period (2005-2017). The antimicrobial susceptibility was tested against nine antibacterial agents using the broth microdilution method. Neighbor-joining tree was constructed to establish the phylogenetic relationships among isolates.

Results: We found that all ureaplasma isolates were resistant to ciprofloxacin and erythromycin, intermediately resistant to azithromycin, and susceptible to doxycycline, moxifloxacin and josamycin. Ofloxacin and levofloxacin resistance was found in 73.27 and $17.82 \%$, respectively, while $37.62 \%$ of isolates proved resistant to tetracycline. Consequently, we detected an elevated multidrug resistance rate among ureaplasma isolates (37.62\%), particularly among serovars 2, 5, 8, and 9 (77.77\% overall), as well as serovars 4, 10, 12, and 13 (52.63\% overall). In most cases, drug resistance was found to be associated with known molecular mechanisms, yet we have identified two novel mutations in the L22 protein, which might be associated with macrolide-resistance.
\end{abstract}

Conclusion: To our knowledge, this is the first study that reports the widespread expansion of multidrug resistance among Ureaplasma serovars, a finding of importance in terms of both surveillance and antimicrobial usage.

Keywords: Ureaplasma spp., Biotyping, Serovars, Multidrug resistance, Tetracyclines, Fluoroquinolones, L22 mutation, Infertility

\section{Introduction}

Ureaplasma spp. are members of the Mollicutes class of bacteria including Ureaplasma parvum (UPA) and Ureaplasma urealyticum (UUR). They are recognized as one of the smallest known self-replicating and free-living organisms. They are common inhabitants of the human genital tract, which colonization could reach $80 \%$ of healthy women in some areas of the world [1]. By contrast, ureaplasmas are less frequently isolated in the lower urogenital tract of healthy men (approximately 20-29\%) [2]. The socioeconomic status, sexual activity with multiple partners, menstrual cycle, pregnancy, the use of vaginal contraceptives, and age, as well as bacterial and protozoan co-

\footnotetext{
* Correspondence: boutheina.mardassi@pasteur.tn

${ }^{1}$ Group of Mycoplasmas, Laboratory of Molecular Microbiology, Vaccinology, and Biotechnology Development, Institut Pasteur de Tunis, Université de Tunis El Manar, 13, Place Pasteur-B.P 74, 1002 Tunis-Belvédère, Tunisia Full list of author information is available at the end of the article
}

infections, favour the colonization of women genital tract by ureaplasmas [3]. Genital ureaplasmas are thought to induce a wide spectrum of pathological conditions in both men and women, including for exemple urethritis, endometritis, chronic prostatitis and bacterial vaginosis [3]. Ureaplasma spp. infections are undoubtedly associated with an increased risk of adverse pregnancy outcomes such as miscarriage, stillbirth, chorioamnionitis, and preterm labor [4]. They also appear to have an etiological role in a variety of severe infections in newborns such as pneumonia, bacteremia, meningitis, and chronic lung disease [5]. In addition, several investigators have presented them as causal agents of infertility [6]. In Tunisia, the role of ureaplasmas in reproductive performance impairment has been reported [7].

Currently, Ureaplasma spp. include 14 serovars, which are grouped into two independent species, designated UPA

(c) The Author(s). 2020 Open Access This article is distributed under the terms of the Creative Commons Attribution 4.0 International License (http://creativecommons.org/licenses/by/4.0/), which permits unrestricted use, distribution, and 
(serovars 1, 3, 6 and 14) and UUR (serovars 2, 4, 5 and 713). It has been speculated for many years that individual ureaplasma species or serovars may be associated with certain diseases more than others. Although several studies have reported that UUR is more pathogenic than UPA [8$10]$, conflicting results were found by others [11], so it is possible that differential pathogenicity may exist at the serovar level rather than at the species level. For example, serovar 3 was more frequently isolated from genital tract infections [7]. Similary, it has been suggested that different serovars could be responsible for different responses to antimicrobial agents [12].

Since ureaplasmas lack a cell wall structure, antibacterial agents like glycopeptides and $\beta$-lactams are completely ineffective. Likewise, ureaplasmas also have natural resistance to lincosamides (e.g. clindamycin) [13]. Hence, drugs for treatment of ureaplasma infections are confined primarily to bacteriostatic agents such as protein synthesis inhibitors (tetracyclines and macrolides), as well as, bactericidal agents that inhibit DNA replication (fluoroquinolones). As a consequence of the inappropriate use of all of the above-mentioned antibiotic classes, acquired resistance of ureaplasma isolates to these antibiotics has been documented in several studies with a rising trend, especially in developing countries [14]. Such a situation, could lead to the emergence of multidrug-resistant (MDR) strains, defined as those resistant to at least one agent in three or more antimicrobial classes [15], thus considerably restricting the treatment options. MDR Mycoplasma strains have been recently reported, particularly in $\mathrm{Myco-}$ plasma genitalium [16], while such findings are still relatively scarce in ureaplasmas [6].

The extent of antimicrobial resistance varies geographically, depending on the different antimicrobial therapy policies and the history of prior use of antimicrobial agents. To our knowledge, data regarding ureaplasma antibiotic susceptibility in Tunisia are scarce and local statistics are particularly needed to establish effective treatment. Therefore, the aim of the present study is threefold: (i) to determine the prevalence of Ureaplasma serovars isolated from Tunisian patients suffering from gynecological infections or infertility, (ii) to study their susceptibility patterns to various antimicrobial agents, and (iii) to investigate the underlying mechanisms of resistance.

\section{Material and methods}

\section{Clinical specimens}

Between years 2005 and 2017, a total of 1057 specimens from patients from Grand Tunis (in the Northeast of Tunisia) were examined by the Laboratory of Mycoplasmas of the Institut Pasteur de Tunis. The clinical specimens consisted of vaginal swabs and semen samples from patients presenting gynecological infections or infertility. The reference strains Ureaplasma parvum serovar 3 (UPA3)
ATCC 27815, and Ureaplasma urealyticum serovar 8 (UUR8) ATCC 27618 were included in this study.

\section{Ureaplasma spp. isolation}

All specimens were tested for the presence of Ureaplasma spp. by SP4-U broth and solid medium, as described by Ben Abdelmoumen Mardassi et al. [7]. As mycoplasma cultures obtained from clinical specimens can represent a mixture of several species, cloning was undertaken as detailed elsewhere [17]. Briefly, positive samples were cloned three times from single colonies recovered after plating of several dilutions.

\section{DNA extraction, species identification and serotyping of Ureaplasma spp.}

Samples were prepared for PCR amplifications as described by kong et al. [18]. Briefly, cells from $2 \mathrm{ml}$ of logarithmicphase culture of each isolate were harvested by centrifugation (Heraeus Megafuge 8R, Thermo Fisher Scientific, USA) at 24,000 X $g$ for $20 \mathrm{~min}$. DNA was isolated by treatment with $200 \mu \mathrm{l}$ of digestion buffer $(10 \mathrm{mM}$ Tris- $\mathrm{HCl}, \mathrm{pH}$ 8.0; $0.45 \%$ Tween 20) and proteinase $\mathrm{K}(100 \mu \mathrm{g} / \mathrm{ml})$. Lysates were incubated for $1 \mathrm{~h}$ at $60^{\circ} \mathrm{C}$ and then for 10 min at $100{ }^{\circ} \mathrm{C}$, and finally stored at $-20^{\circ} \mathrm{C}$. The primer sequences of the genes used in this study are displayed in Additional file 1: Table S1. Oligonucleotide primers UMS125/UMA226 were used for amplification of the $5^{\prime}$-end of $m b a$ (Multiple Banded Antigen) gene for species identification. All amplifications were performed in a final volume of $50 \mu$ consisted of 1 X PCR buffer $(10 \mathrm{mM}$ Tris- $\mathrm{HCl}, \mathrm{pH}$ 8.3; $50 \mathrm{mM} \mathrm{KCl}$ ) (Invitrogen, USA), $2.5 \mathrm{mM} \mathrm{MgCl}_{2}$ (Invitrogen, USA), $0.4 \mu \mathrm{M}$ of each primer (Sigma Genosys, Germany), $0.2 \mathrm{mM}$ dNTPs (Sigma-Aldrich, Germany), 1.25 units of Taq DNA polymerase (Invitrogen, USA) and $10 \mu \mathrm{l}$ of treated sample. An Applied Biosystems Thermal Cycler 2720 (Life Technologies, USA) was set up with a first cycle of denaturation for $5 \mathrm{~min}$ at $94{ }^{\circ} \mathrm{C}$, followed by 35 cycles of denaturation at $94{ }^{\circ} \mathrm{C}$ for $1 \mathrm{~min}$, annealing at $60{ }^{\circ} \mathrm{C}$ for $1 \mathrm{~min}$, elongation at $72{ }^{\circ} \mathrm{C}$ for $1 \mathrm{~min}$, and a final extension step of $7 \mathrm{~min}$ at $72{ }^{\circ} \mathrm{C}$. The PCR products were electrophoresed on $2 \%$ agarose gel and stained with ethidium bromide (Sigma-Aldrich, Germany) to visualize the DNA bands, whose sizes were determined using a 100-bp DNA ladder (Invitrogen, USA). Based on the amplicon size, Ureaplasma strains were assigned to UPA (403 or $404 \mathrm{bp}$ ) or UUR (448 bp) [19]. The PCR products of the 5 '-end of $m b a$ gene of all strains were sequenced for serotyping characterization of Ureaplasma serovars. Briefly, PCR products were purified with both Exonuclease I (Biolabs, England) and Shrimp Alkaline Phosphatase (Biolabs, England) as per manufacturer's instructions. Sequencing reactions were performed using a BigDye Terminator v3.1 cycle sequencing kit (Applied Biosystems, USA), according to the manufacturer's recommendations, and were run in 
an ABI Prism 3130 genetic analyzer (Applied Biosystems, USA). Both strands from each PCR amplicon were sequenced twice. The nucleotide sequences obtained were aligned with reference sequences of 14 ATCC Ureaplasma serovars, that were retrieved from GenBank as follows: ABES01000000 (UPA1), ABFL02000000 (UUR2), CP00094 2.1 (UPA3), AAYO02000000 (UUR4), AAZR01000000 (UU R5), AAZQ01000000 (UPA6), AAYP01000000 (UUR7), AA YN02000000 (UUR8), AAYQ02000000 (UUR9), CP001184 (UUR10), AAZS01000000 (UUR11), AAZT01000000 (UUR 12), ABEV01000000 (UUR13) and ABER01000000 (UPA1 4). The alignment was conducted by using BioEdit Sequence Alignment Editor version 7.2.5 [20].

\section{Phylogenetic analysis}

The $5^{\prime}$-end of $m b a$ gene sequences of all clinical isolates (403/404 bp for UPA and 448 bp for UUR) were phylogenetically analyzed by using MEGA version 6.06 [21]. The evolutionary distances were estimated by using Kimura's two-parameter method, and a circular phylogenetic tree was constructed using the neighbor-joining method. Bootstrap analyses were performed by 1000 resamplings of the data sets.

\section{Antimicrobial agents}

The susceptibility tests included nine antimicrobial agents: tetracyclines (tetracycline 'TET', doxycycline 'DOX'); fluoroquinolones (ofloxacin 'OFX', ciprofloxacin 'CIP', levofloxacin 'LVX', moxifloxacin 'MXF'); and macrolides (erythromycin 'ERY', azithromycin 'AZM' and josamycin 'JOS') (Sigma-Aldrich, Germany). Each antimicrobial agent was prepared according to the manufacturer's instructions at a stock concentration of 1280 $\mathrm{mg} / \mathrm{L}$ and stored at $-20^{\circ} \mathrm{C}$ before use.

\section{Antimicrobial susceptibility testing}

Antimicrobial susceptibility was determined using a broth microdilution method following the Clinical Laboratory Standards Institute (CLSI) guidelines [22]. The reference type strains UPA3 ATCC 27815 and UUR 8 ATCC 27618 were included in each test to ensure quality control. The specific breakpoints $(\mathrm{mg} / \mathrm{L})$ indicating susceptibility $(S)$ or resistance $(R)$ are: tetracycline $S \leq 1$, $R \geq 2$; doxycycline $S \leq 4, R \geq 8$; ciprofloxacin $S \leq 1, R \geq 2$; ofloxacin $\mathrm{S} \leq 1, \mathrm{R} \geq 4$; levofloxacin $\mathrm{S} \leq 2, \mathrm{R} \geq 4$; moxifloxacin $S \leq 2, R \geq 4$; erythromycin $S \leq 8, R \geq 16$; azithromycin $S \leq 0.125, R \geq 4$; and josamycin $S \leq 2, R \geq 8$. Since interpretative criteria for DOX, OFX, CIP, JOS, and AZI are not available from CLSI, the minimum inhibitory concentration (MIC) values were interpreted according to the Mycoplasma IST-2 kit (bioMerieux, Marcyl'Etoile, France) criteria.

\section{Resistance genes amplification and sequencing}

tet $(\mathrm{M})$ and Int-Tn genes were amplified from both susceptible- and resistant-strains as previously detailed [16]. For fluoroquinolone-resistant strains, the quinolone resistance determining regions (QRDRs) of $g y r A$, gyrB, parC, and parE genes were studied by PCR amplification and sequencing [23]. Simultaneously, macrolide-resistant strains underwent PCR amplification and sequencing of both $23 \mathrm{~S}$ rRNA alleles, as well as the genes encoding ribosomal proteins L4 and L22 [24]. Amplicons were then purified and sequenced as detailed above. Sequences were analyzed using BioEdit and translated into amino acids (http://www.expasy.org/). The amino acid sequences of GyrA, GyrB, ParC, ParE, L4, and L22 proteins, as well as the nucleotide sequences of the $23 \mathrm{~S}$ rRNA genes were compared with the respective sequences of the 14 ATCC Ureaplasma serovars, in order to distinct species- or serovar-specific polymorphisms from those associated with resistance.

\section{Statistical analyses}

Statistical analyses were conducted using GraphPad Prism for Windows v. 7.03. The statistical tests applied in this study were the Chi-Square test and t-test. A $P$ value $<0.05$ was considered as statistically significant.

\section{Results}

Prevalence of Ureaplasma spp. in the urogenital tract Ureaplasma spp. were detected in high titers $\left(\geq 10^{4} \mathrm{CFU} /\right.$ $\mathrm{ml})$ in $101(9.55 \%)$ out of 1057 cultured samples. Out of these, 85 isolates $(84.2 \%)$ were recovered from infertile patients and 16 strains (15.8\%) from patients suffering from gynecological infections (Additional file 1: Table S2).

All specimens found to be positive for Ureaplasma spp. were biotyped by $m b a$-based PCR. Of the 101 Ureaplasma isolates, UPA and UUR were found in 73 (72.28\%) and 28 (27.72\%) patients, respectively. None of the patients was coinfected with both species. UPA- and UUR- positive isolates were further subtyped into different serovars by nucleotide sequencing. Among UPA strains, serovar 3 was the most frequent ( 35 out of $73,47.94 \%$ ), followed by serovars 1 and 6 (19 out of 73 for each one, 26.03\%). Subtyping of UUR from the 28 positive cases showed serovars 4 , 10,12 , and 13 in 19 cases (67.86\%) and serovars 2, 5, 8 and 9 in 9 cases (32.14\%). No UPA serovar 14 or UUR serovars 7 and 11 were detected (Additional file 2: File S1, File S2, File S3, File S4 and File S5).

\section{Correlation between Ureaplasma serovars and gender}

The positive rate of ureaplasma infections was higher in female, who were in the reproductive age (25-40 years old) (76 cases), than male (25 cases). By contrast, the distribution of different Ureaplasma serovars showed no 
statistically significant difference between the two genders $(P=0.7835)$.

\section{Correlation between Ureaplasma serovars and clinical manifestations}

The positive rate of Ureaplasma spp. was higher in infertile patients (85 of 101, 84.2\%) then patients suffering from gynecological infections (16 of 101, $15.8 \%)$. The distribution of Ureaplasma serovars among patients with different clinical status showed a statistically significant difference $(P=0.0102)$. In patients with genital tract infections, serovar 1 was predominant in 8 cases (50\%) while in infertile patients, serovar 3 was predominant in 29 cases (34.12\%). Interestingly, we observed that UUR $2,5,8,9$, and UUR 4, 10, 12, 13 clinical isolates were exclusively recovered from infertile patients.

\section{Antimicrobial susceptibility patterns over the past 12 years}

A summary of the MIC results of the 101 Ureaplasma spp. clinical isolates is shown in Additional file 1: Table S3.

When comparing MIC data between species, UUR isolates had significantly higher MIC values compared to UPA isolates against DOX, OFX, CIP, LVX, MXF, and ERY (Fig. 1). By contrast, no significant difference between the two species was observed in the MICs of TET, AZM, and JOS $(P>0.05)$.

Regardless to serovars, statistical analysis showed a significant difference in the susceptibility rates of Ureaplasma spp. strains to different antibacterial agents $(P<$ 0.0001). Indeed, all Ureaplasma spp. isolates were: sensitive to DOX, MXF, and JOS (MICs $\leq 1 \mathrm{mg} / \mathrm{L}$ ); moderately sensitive to AZM $(0.25 \leq \mathrm{MICs} \geq 2 \mathrm{mg} / \mathrm{L})$; and resistant to ERY (MICs $\geq 16 \mathrm{mg} / \mathrm{L}$ ) and CIP (MICs $\geq 4$ $\mathrm{mg} / \mathrm{L})$. Regarding tetracycline susceptibility, 63 (62.38\%)

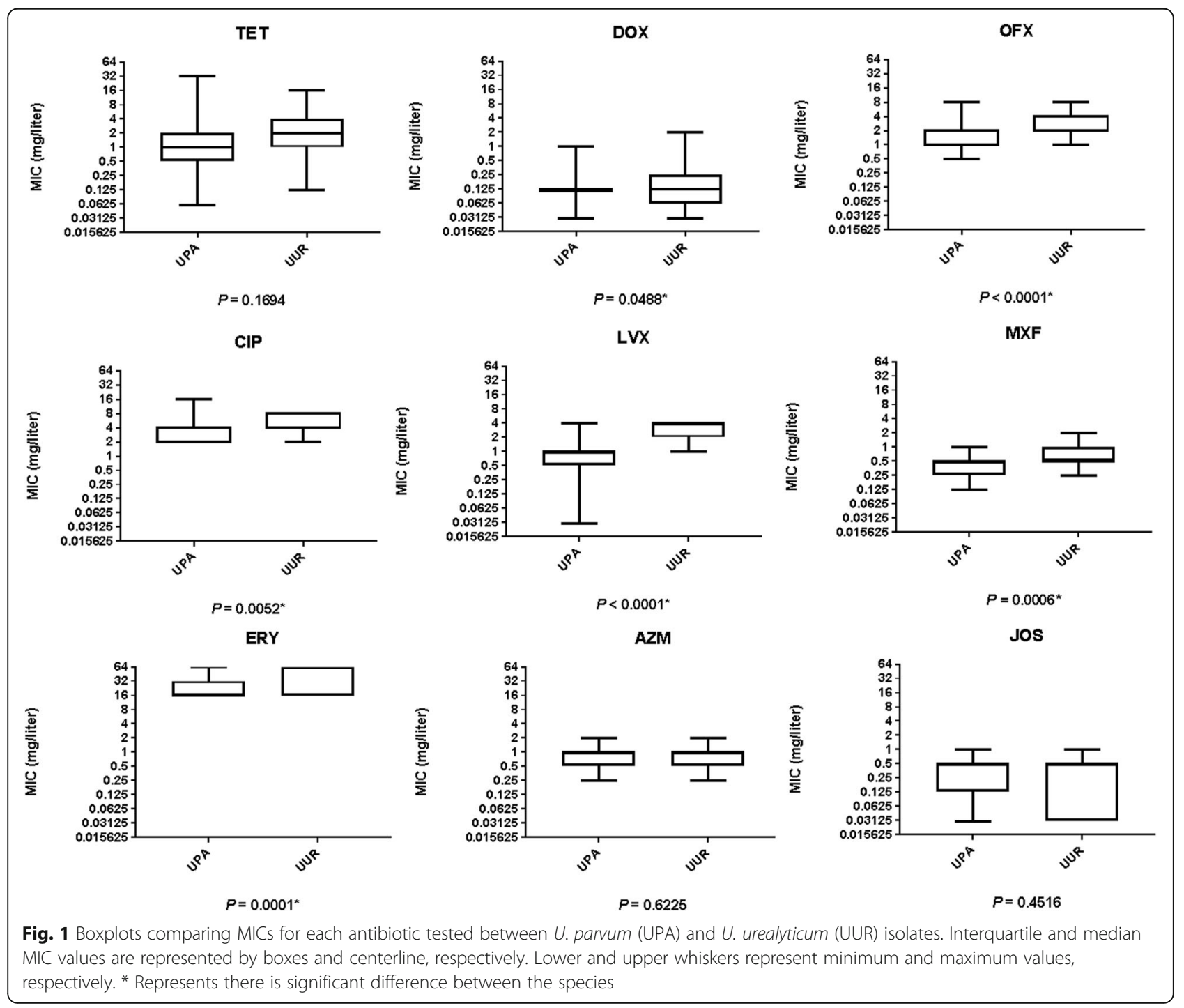


strains were found sensitive. The examination of susceptibility to fluoroquinolones indicated that 27 (26.73\%) strains were sensitive and 35 (34.65\%) were moderately sensitive to OFX. Yet, Ureaplasma spp. strains showed a high sensitivity rate to LVX (83/101, 82.18\%) (Fig. 2). In addition, we noticed that the susceptibility rate to TET, OFX and LVX remained almost constant throughout the studied time period $(P=0.1917)$.

Sex-, clinical status-, and serovar-specific trends in antibiotic resistance patterns of Ureaplasma spp.

Compared with strains isolated from male patients, strains isolated from female patients displayed slightly higher resistance rates to LVX and OFX, as well as slightly lower resistance rate to TET.

Strains isolated from infertile patients displayed a significant higher resistance rate to TET and a significant higher sensitivity rate to OFX compared to those isolated from patients presenting with genital infections (Additional file 1: Table S4). However, the observed difference in resistance rates to LVX between the two clinical manifestations was not statistically significant.

Interestingly, Ureaplasma serovars showed a significant difference in their resistance patterns to TET, OFX, and LVX $(P<0.0001)$ (Fig. 3). Indeed, UUR strains showed more resistance to TET, OFX, and LVX (60.71, 85.71, and $64.29 \%$, respectively) than did UPA strains $(28.77,20.55$, and $0 \%$, respectively) $(P<0.0001)$. UUR2, 5, 8, 9 strains displayed the highest resistance rates to TET (77.77\%), OFX (100\%) and LVX (88.88\%). However, UPA6 strains were the most sensitive with a resistance rate of 5.26, 5.26, and $0 \%$ to TET, OFX, and LVX, respectively.

\section{Clustering of multidrug-resistant strains}

The NJ tree based on the 5 -end sequence of $m b a$ gene clearly distinguished five clusters, which corresponded to UPA3, UPA6, UPA1, UUR2, 5, 8, 9 and UUR4, 10, 12, 13 (Fig. 4). Overall, $37.62 \%$ of the strains were MDR, with $60.71 \%(17 / 28)$ of UUR strains and $28.76 \%(21 / 73)$ of UPA being MDR. Regarding serovars, the majority of UUR2, 5, 8, $9(77.77 \%, 7 / 9)$ were MDR followed by UUR4, 10, 12, 13 (52.63\%, 10/19), and UPA3 (48.57\%, $17 / 35)$. However, $15.79 \%$ of UPA1 (3/19) and only one isolate of UPA6 were MDR.

\section{Molecular analysis of genetic mechanisms of resistance Tetracyclines}

Screening of the tet $(\mathrm{M})$ determinant, which is associated with tetracycline resistance, as well as the Int-Tn gene, revealed that all tetracycline-resistant Ureaplasma spp. clinical isolates, simultaneously, harboured these two genes (Additional file 1: Figure S5). All the PCR products of tet $(\mathrm{M})$ and Int-Tn genes migrated at the expected sizes of $397 \mathrm{bp}$ and $579 \mathrm{bp}$, respectively. Neither tet $(\mathrm{M})$ nor Int-Tn sequences could be amplified from tetracycline-sensitive clinical isolates.

\section{Fluoroquinolones}

The nucleotide and deduced amino acid sequences of the QRDRs of $\operatorname{gyr} A, \operatorname{gyr} B, \operatorname{parC}$ and parE for a selection of clinical Ureaplasma spp. strains were compared with the respective 14 ATCC Ureaplasma serovars, and thus species- or serovar-specific polymorphisms could be avoided.

Sequence comparison revealed no mutations in any of the sequenced isolates within either $\operatorname{gyr} A$ or $g y r B$ genes; however, mutations were observed in the parC and parE genes. A single base pair change (C248T) in the parC gene was identified in all levofloxacin-resistant strains, resulting in the substitution of serine by leucine at position 83 (S83L) (Additional file 2: File S6). A point mutation at position $1336(\mathrm{C} 1336 \mathrm{~T})$ in the parE gene was identified in three levofloxacin- resistant strains, resulting in the proline-to-serine substitution at position 446 (P446S) (Additional file 2: File S7). For the above clinical strains, which harboured the double mutation S83L in ParC and P446S in ParE, the MICs of the four fluoroquinolones were higher (OFX: $8 \mathrm{mg} / \mathrm{L}, \mathrm{CIP}: 8 \mathrm{mg} / \mathrm{L}$, LVX: 4 mg/L, MXF: $2 \mathrm{mg} / \mathrm{L}$ ) compared to strains bearing the

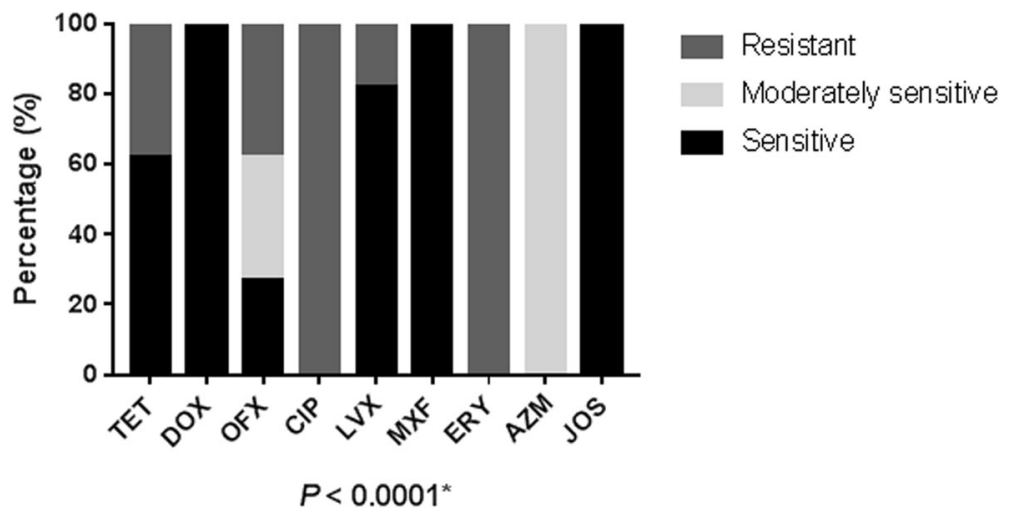

Fig. 2 Antimicrobial susceptibility of Ureaplasma spp. strains to the antibacterial agents. ${ }^{*}$ Represents there is significant difference 


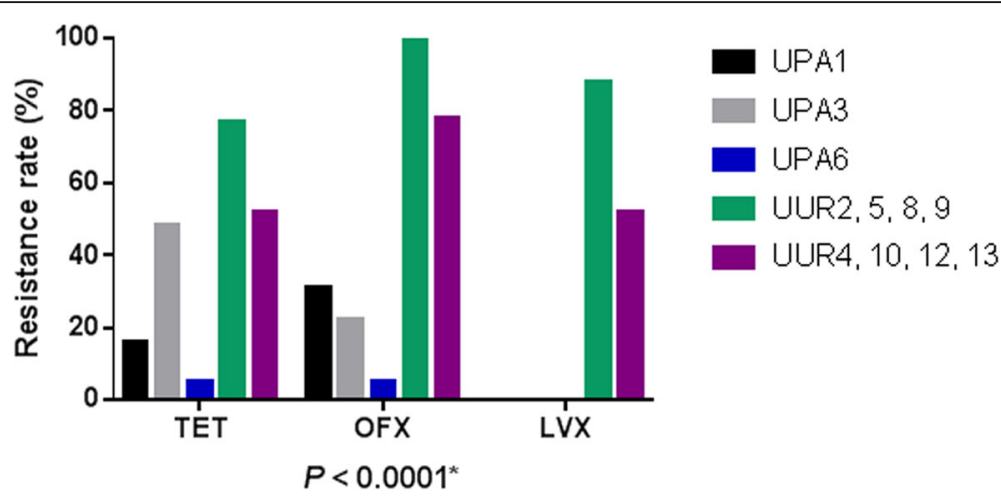

Fig. 3 Antibiotic resistance rates of Ureaplasma serovars to TET, OFX, and LVX. ${ }^{*}$ Represents there is significant difference among the serovars

single mutation S83L in ParC. However, no mutation was detected in four ciprofloxacin-resistant strains (MIC: $4 \mathrm{mg} / \mathrm{L}$ ). Detailed results of the genes responsible for antimicrobial resistance are summarized in Table 1.

\section{Macrolides}

The 23S rRNA genes and associated L4 and L22 proteins were sequenced from 26 ureaplasma isolates resistant to ERY (MICs $\geq 16 \mathrm{mg} / \mathrm{L}$ ) (Table 1 ). No mutations were found in either the 23S rRNA operons or the L4 protein of any of the isolates, yet two new point mutations (G361
$\mathrm{T}$ and $\mathrm{C} 422 \mathrm{~T}$ ) were detected in the $\mathrm{L} 22$ gene of all isolates studied that translated into amino acid substitutions A121S and T141I, respectively (Additional file 2: File S8).

\section{Discussion}

Compared to the rate of multidrug resistance recently reported by Zhu et al. [6], our study is reporting a higher rate among clinical strains of ureaplasmas. Indeed, the prevalence of MDR Ureaplasma spp. strains tested herein was particularly high (37.62\%), which is likely to be the result of the widespread uncontrolled and

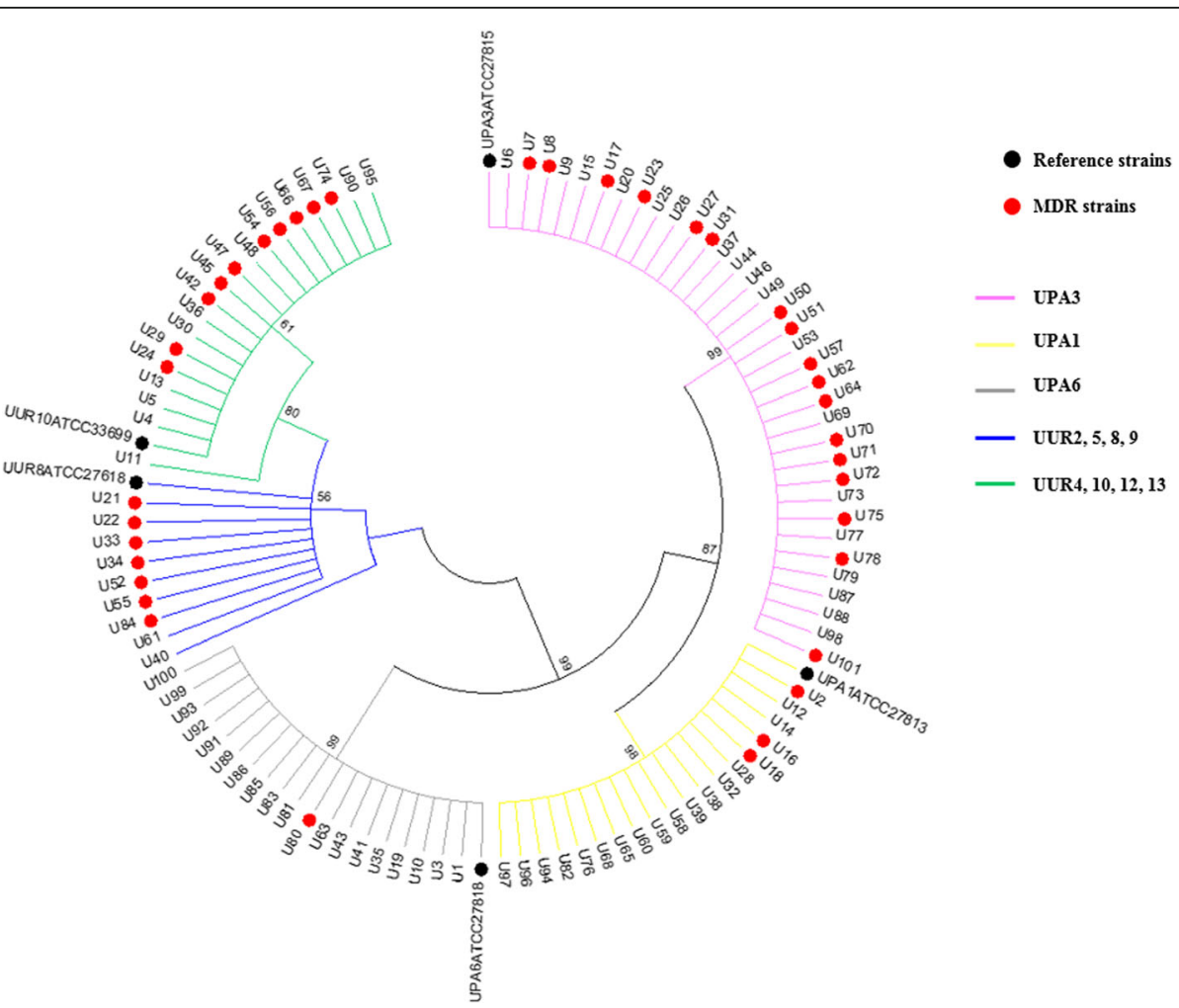

Fig. 4 Neighbor-joining tree of 101 Ureaplasma spp. isolates based on the $5^{\prime}$-end of mba gene sequences. The branches are colored according to the serovar. Black circles correspond to reference strains and MDR isolates are represented by red circles 
Table 1 Molecular characterization of antibiotic resistance mechanisms of 26 Ureaplasma spp. isolates

\begin{tabular}{|c|c|c|c|c|c|c|c|c|c|c|c|}
\hline \multirow[t]{2}{*}{ Strain } & \multirow[t]{2}{*}{ Serovar } & \multicolumn{7}{|c|}{ MICs (mg/liter) $)^{a}$} & \multicolumn{2}{|c|}{ QRDRs analysis $^{\mathrm{b}}$} & \multirow{2}{*}{$\begin{array}{l}\text { Macrolide resistance trait } \\
\text { L22 }\end{array}$} \\
\hline & & OFX & CIP & LVX & MXF & AZM & ERY & JOS & ParC & ParE & \\
\hline ATCC 27815 & UPA3 & $0.25(\mathrm{~S})$ & $0.5(S)$ & $0.125(S)$ & $0.06(S)$ & $0.06(\mathrm{~S})$ & $2(S)$ & $0.03(\mathrm{~S})$ & WT & WT & WT \\
\hline ATCC 27618 & UUR8 & $0.5(S)$ & $0.5(S)$ & $0.25(\mathrm{~S})$ & $0.06(S)$ & $0.06(S)$ & $2(S)$ & $0.03(\mathrm{~S})$ & WT & WT & WT \\
\hline U1 & UPA6 & 2 (MS) & $8(R)$ & $1(\mathrm{~S})$ & $0.5(\mathrm{~S})$ & 1 (MS) & $64(\mathrm{R})$ & $0.5(S)$ & $\mathrm{S} 83 \mathrm{~L}$ & WT & A121S, T141I \\
\hline U2 & UPA1 & 2 (MS) & $4(\mathrm{R})$ & $1(S)$ & $0.5(\mathrm{~S})$ & 0.5 (MS) & $16(\mathrm{R})$ & $0.5(S)$ & WT & WT & A121S, T141I \\
\hline U4 & UUR4, 10, 12, 13 & 2 (MS) & $4(\mathrm{R})$ & $2(S)$ & $0.5(\mathrm{~S})$ & 1 (MS) & $64(\mathrm{R})$ & $0.03(\mathrm{~S})$ & WT & WT & A121S, T141I \\
\hline U6 & UPA3 & $4(R)$ & $4(\mathrm{R})$ & $0.25(\mathrm{~S})$ & $0.5(S)$ & 0.25 (MS) & $16(\mathrm{R})$ & $0.125(\mathrm{~S})$ & WT & WT & A121S, T141I \\
\hline U10 & UPA6 & $4(\mathrm{R})$ & $8(R)$ & $1(\mathrm{~S})$ & $0.5(S)$ & 0.5 (MS) & $16(\mathrm{R})$ & $0.5(\mathrm{~S})$ & $\mathrm{S} 83 \mathrm{~L}$ & WT & A121S, T141I \\
\hline U11 & UUR4, 10, 12, 13 & $4(R)$ & $4(R)$ & $4(R)$ & $1(\mathrm{~S})$ & 1 (MS) & $32(\mathrm{R})$ & $0.03(\mathrm{~S})$ & $\mathrm{S} 83 \mathrm{~L}$ & WT & A121S, T141I \\
\hline U13 & UUR4, 10, 12, 13 & $4(\mathrm{R})$ & $8(R)$ & $4(\mathrm{R})$ & $0.5(S)$ & 0.25 (MS) & $64(\mathrm{R})$ & $0.03(S)$ & $\mathrm{S} 83 \mathrm{~L}$ & WT & A121S, T141I \\
\hline U14 & UPA1 & $8(R)$ & $16(\mathrm{R})$ & $1(S)$ & $0.5(S)$ & 2 (MS) & $64(\mathrm{R})$ & $0.5(S)$ & $\mathrm{S} 83 \mathrm{~L}$ & WT & A121S, T141I \\
\hline U15 & UPA3 & $8(\mathrm{R})$ & $8(R)$ & $0.5(\mathrm{~S})$ & $0.5(S)$ & 1 (MS) & $16(\mathrm{R})$ & $0.5(S)$ & $\mathrm{S} 83 \mathrm{~L}$ & WT & A121S, T141I \\
\hline U16 & UPA1 & $1(\mathrm{~S})$ & $4(R)$ & $0.5(S)$ & $0.25(\mathrm{~S})$ & $0.5(\mathrm{MS})$ & $16(\mathrm{R})$ & $0.125(\mathrm{~S})$ & WT & WT & A121S, T141I \\
\hline U21 & UUR2, 5, 8, 9 & $8(\mathrm{R})$ & $8(\mathrm{R})$ & $4(\mathrm{R})$ & $2(S)$ & 1 (MS) & $64(\mathrm{R})$ & $0.25(\mathrm{~S})$ & $\mathrm{S} 83 \mathrm{~L}$ & P446S & A121S, T141I \\
\hline U22 & UUR2, 5, 8, 9 & $4(\mathrm{R})$ & $4(R)$ & $4(\mathrm{R})$ & $1(\mathrm{~S})$ & 2 (MS) & $16(\mathrm{R})$ & $0.5(S)$ & $\mathrm{S} 83 \mathrm{~L}$ & WT & A121S, T141I \\
\hline U24 & UUR4, 10, 12, 13 & $4(\mathrm{R})$ & $4(\mathrm{R})$ & $4(\mathrm{R})$ & $0.5(\mathrm{~S})$ & 1 (MS) & $64(\mathrm{R})$ & $0.03(\mathrm{~S})$ & $\mathrm{S} 83 \mathrm{~L}$ & WT & A121S, T141I \\
\hline U30 & UUR4, 10, 12, 13 & $4(\mathrm{R})$ & $8(R)$ & $4(\mathrm{R})$ & $0.5(S)$ & 1 (MS) & $32(\mathrm{R})$ & $0.03(\mathrm{~S})$ & $\mathrm{S} 83 \mathrm{~L}$ & WT & A121S, T141I \\
\hline U33 & UUR2, 5, 8, 9 & $4(\mathrm{R})$ & $4(\mathrm{R})$ & $4(\mathrm{R})$ & $0.5(S)$ & 1 (MS) & $64(\mathrm{R})$ & $1(\mathrm{~S})$ & S83L & WT & A121S, T141I \\
\hline U34 & UUR2, 5, 8, 9 & $4(\mathrm{R})$ & $8(R)$ & $4(\mathrm{R})$ & $0.5(S)$ & $0.5(\mathrm{MS})$ & $16(\mathrm{R})$ & $1(\mathrm{~S})$ & $\mathrm{S} 83 \mathrm{~L}$ & WT & A121S, T141I \\
\hline U36 & UUR4, 10, 12, 13 & $4(\mathrm{R})$ & $8(R)$ & $4(R)$ & $0.5(S)$ & 0.25 (MS) & $64(\mathrm{R})$ & $0.03(\mathrm{~S})$ & $\mathrm{S} 83 \mathrm{~L}$ & WT & A121S, T141I \\
\hline U40 & UUR2, 5, 8, 9 & $4(R)$ & $4(\mathrm{R})$ & $4(R)$ & $0.5(S)$ & 0.5 (MS) & $64(\mathrm{R})$ & $0.5(\mathrm{~S})$ & $\mathrm{S} 83 \mathrm{~L}$ & WT & A121S, T141I \\
\hline U47 & UUR4, 10, 12, 13 & $4(R)$ & $4(\mathrm{R})$ & $4(\mathrm{R})$ & $1(\mathrm{~S})$ & 0.5 (MS) & $16(\mathrm{R})$ & $0.5(S)$ & $\mathrm{S} 83 \mathrm{~L}$ & WT & A121S, T141I \\
\hline U52 & UUR2, 5, 8, 9 & $4(R)$ & $8(R)$ & $4(R)$ & $0.5(S)$ & 1 (MS) & $64(\mathrm{R})$ & $0.5(S)$ & $\mathrm{S} 83 \mathrm{~L}$ & WT & A121S, T141I \\
\hline U54 & UUR4, 10, 12, 13 & $4(\mathrm{R})$ & $8(R)$ & $4(\mathrm{R})$ & $1(\mathrm{~S})$ & 2 (MS) & $64(\mathrm{R})$ & $0.5(S)$ & $\mathrm{S} 83 \mathrm{~L}$ & WT & A121S, T141I \\
\hline U55 & UUR2, 5, 8, 9 & $8(\mathrm{R})$ & $8(R)$ & $4(\mathrm{R})$ & $2(S)$ & 2 (MS) & $64(\mathrm{R})$ & $0.5(\mathrm{~S})$ & S83L & P446S & A121S, T141I \\
\hline U56 & UUR4, 10, 12, 13 & $4(R)$ & $4(R)$ & $4(R)$ & $1(\mathrm{~S})$ & 2 (MS) & $64(\mathrm{R})$ & $0.25(S)$ & $\mathrm{S} 83 \mathrm{~L}$ & WT & A121S, T141I \\
\hline U66 & UUR4, 10, 12, 13 & $4(\mathrm{R})$ & $4(R)$ & $4(\mathrm{R})$ & $1(\mathrm{~S})$ & $0.5(\mathrm{MS})$ & $16(R)$ & $0.5(S)$ & $\mathrm{S} 83 \mathrm{~L}$ & WT & A121S, T141I \\
\hline U67 & UUR4, 10, 12, 13 & $4(R)$ & $4(\mathrm{R})$ & $4(R)$ & $1(\mathrm{~S})$ & 2 (MS) & $64(\mathrm{R})$ & $0.25(\mathrm{~S})$ & $\mathrm{S} 83 \mathrm{~L}$ & WT & A121S, T141I \\
\hline U84 & UUR2, 5, 8, 9 & $8(\mathrm{R})$ & $8(R)$ & $4(\mathrm{R})$ & $2(S)$ & 1 (MS) & $64(\mathrm{R})$ & $0.5(S)$ & $\mathrm{S} 83 \mathrm{~L}$ & P446S & $\mathrm{A} 121 \mathrm{~S}, \mathrm{~T} 141 \mathrm{I}$ \\
\hline
\end{tabular}

${ }^{a} S$ Sensitive, MS Moderately sensitive, $R$ Resistant, ${ }^{b} W T$ Wild-type

increased misuse of antibacterial agents in Tunisia, a phenomenon that has been previously stressed $[25,26]$.

In line with previous reports, this study showed higher MIC trends exhibited by UUR compared to UPA [27, 28], suggesting a possible inherently increased tolerance of UUR isolates to drugs relative to that of UPA isolates, particularly serovars 2, 5, 8, 9 . However, differences in susceptibilities, with sometimes conflicting results, have been reported between the two ureaplasma species and within the 14 distinct serovars [12]. Unexpectedly, we found an elevated multi-drug resistance rate in UPA3. Given that all the isolates were uniformely resistant to CIP and ERY, this result could be explained by the high resistance rate of UPA3 to TET.
Tetracyclines represent the first-line treatment of infections caused by Ureaplasma spp., to which resistance is continuously increasing. Herein, Ureaplasma spp. strains showed a reduced susceptibility to TET (62.38\%), but an extremely high sensitivity to DOX, as reported previously [29]. This rate is fairly consistent with that reported by Díaz et al. in Cuba [30], but distinctly different to a study from France, which reported a higher (>80\%) sensitivity rates to TET [31].

High percentages of resistance to fluoroquinolones was demonstrated, most likely as a consequence of their more frequent use for plenty of infections. Hitherto, it was widely reported that CIP and OFX were mainly inactive [14]. However, the newer fluoroquinolones, LVX and MXF presented a greater effectiveness against 
Ureaplasma spp., as described elsewhere [32]. By contrast, Song et al. have reported in China a higher resistance rate to LVX (76.9\%) and MXF (23.1\%), compared to our findings (17.82 and $0 \%$, respectively) [33]. In our study, all isolates were resistant to ERY and moderately sensitive to AZM, which was surprising as Ureaplasma spp. is known to be susceptible to macrolides [34]. Yet, a study conducted in South Africa has reported a high resistance rate to ERY, reaching 80\% [13]. Similarly, AZMresistant strains of Ureaplasma spp. are now being reported with increasing frequency [12]. No resistance was seen against the new macrolide, JOS for Ureaplasma spp., which is in agreement with earlier studies [14, 29]. Remarkably, the antibacterial resistance profiles of ureaplasma isolates from different geographical locations vary significantly, which might be the result of variations in antimicrobial exposure in different patient populations. In some developing countries, the overuse and/or inappropriate use of antibiotics are relatively common so that resistance patterns may exceed what is seen in the industrialized world [35]. This emphasizes the need for local surveillance of antimicrobial resistance in these countries in order to guide empirical treatment.

Currently, the only known mechanism of tetracycline resistance for Mollicutes is the presence of the tet $(\mathrm{M})$ transferable genetic element. In this study, we demonstrated that our clinical tetracycline-resistant isolates harbored DNA sequences related to the tet $(\mathrm{M})$ determinant and the Int-Tn gene. Previous study conducted in our laboratory has showed that the nucleotide sequence analysis of the tet $(\mathrm{M})$ amplicon revealed a unique sequence shared by all tetracycline-resistant clinical isolates of both UPA and Mycoplasma hominis, suggesting that it may have benefited from horizontal gene transfer, making it highly competent to spread [7].

It seems that fluoroquinolone abuse leads to resistance, mainly through target enzyme mutations. Here, we identified the most common amino acid substitution (S83L) in the ParC protein, which was found in 22 of 26 fluoroquinolone-resistant isolates. This is in agreement with many prior reports indicating that S83L is the most frequently mutation described thus far in Ureaplasma fluoroquinolone-resistant isolates [27]. This mutation is located in the proposed active site of the ParC protein, and is homologous to the mutation identified in other fluoroquinolone-resistant bacteria ( $\mathrm{S} 80 \mathrm{~L})$, such as Acinetobacter baumannii and Pseudomonas aeruginosa [36]. However, we noted that 4 of 26 fluoroquinolone-resistant isolates studied had no amino acid substitutions in the QRDRs. This finding is not without precedent and could be linked to mutations outside the sequenced regions or alternative mechanisms, not yet elucidated, such as altered membrane permeability [37]. An atypical substitution in the QRDR region of the ParE protein, P446S, was detected in only three resistant isolates, which also harbored the S83L substitution. Although P446S was not previously associated with fluoroquinolone resistance in other bacteria, we suggest that it might lead to the increased resistance to fluoroquinolone observed for these three isolates. In agreement to this finding, Chang et al. have recently detected P446S substitution in one ofloxacin-resistant isolate [38]. Interestingly, the same substitution was found also in a highly moxifloxacin-resistant Mycoplasma genitalium strain, which harboured also S83L substitution in ParC protein [39].

With regard to macrolide resistance, we detected two amino acid substitutions in the L22 protein, A121S and T141I, for all the macrolide-resistant strains studied. Both substitutions were previously described in a highly macrolide-resistant strain, but it has been concluded that they may not contribute to macrolide resistance because of the presence of a five-amino acid insertion (69TGKAR70) in the extended loop of the L4 protein, which has been also described in Streptococcus pneumoniae [40]. Yet, additional mutations outside of the investigated regions, DNA methylation, or increased drug efflux may also be responsible for the relative increase in tolerance to erythromycin for these strains. In light of the epidemiological characteristics of ureaplasma strains, the present results may aid Tunisian clinicians to implement rational drug usage that would prevent and control the spread of antibiotic resistance.

\section{Conclusion}

In sum, this study provides a detailed picture on the genetic diversity, drug susceptibility, and resistance mechanisms in Ureaplasma spp. clinical isolates, an investigation that has not previously been drawn up, in Tunisia. Our study shows that Ureaplasma spp. isolation is frequent among infertile patients, and highlights for the first time the emergence of a high rate of MDR strains. Based on these findings, DOX, MXF, and JOS would seem to be effective against ureaplasma infections in Tunisian patients. Thus, the early diagnosis and appropriate treatment of Ureaplasma spp. may prove to be important in reducing infertility in Tunisia. Furthermore, this study reveals two novel mutations in L22 protein that might be associated with macrolide resistance. $\mathrm{Fu}$ ture research are needeed in order to explore the epidemic potential and virulence of Ureaplasma serovars that may be associated with the spread of antimicrobial resistance, particularly MDR strains.

\section{Supplementary information}

Supplementary information accompanies this paper at https://doi.org/10. 1186/s13756-020-0681-5.

Additional file 1: Table S1. Genes and respective flanking oligonucleotide primers used. Table S2. Epidemiologic characteristics of Ureaplasma spp. strains used. Table S3. In-vitro activity of tetracyclines, 
fluoroquinolones, and macrolides against 101 human Ureaplasma spp. isolates. Table S4. Distribution of antimicrobial resistance among patients with genital tract infections and infertility. Figure S5. PCR results screening for tet (M) (A) and Int-Tn genes (B) on 2\% agarose gel. A. MW: GeneRuler 100 base pairs (bp) DNA Ladder. Lane 1: Negative control. Lane 213: Amplicons from the tetracycline-resistant Ureaplasma spp. clinical isolates. Lane 14-15: Amplicons from U. parvum ATCC 27815 and U. urealyticum ATCC 27618. Lane 16-21: Amplicons from tetracycline-sensitive Ureaplasma spp. clinical isolates. The tet $(\mathrm{M})$ expected gene product is 397 bp based on the primers used. B. MW: GeneRuler 100 (bp) DNA Ladder. Lane 1: Negative control. Lane 2: Amplicon from a tetracyclinesensitive Ureaplasma spp. clinical isolate. Lane 3-12: Amplicons from tetracycline-resistant Ureaplasma spp. clinical isolates. The Int-Tn expected gene product is $579 \mathrm{bp}$ based on the primers used. (338 KB).

Additional file 2: File S1. Multiple sequence alignment of the $5^{\prime}$-end of mba gene of the reference strain UPA 1 and 19 Ureaplasma serovar 1 clinical strains. File S2. Multiple sequence alignment of the 5 '-end of mba gene of the reference strain UPA3 and 35 Ureaplasma serovar 3 clinical strains. File S3. Multiple sequence alignment of the $5^{\prime}$-end of mba gene of the reference strain UPA6 and 19 Ureaplasma serovar 6 clinical strains. File S4. Multiple sequence alignment of the $5^{\prime}$-end of mba gene of the reference strains UUR4, UUR10, UUR12, UUR13 and 19 Ureaplasma serovars 4, 10, 12, 13 clinical strains. File S5. Multiple sequence alignment of the $5^{\prime}$-end of mba gene of the reference strains UUR2, UUR5, UUR8, UUR9 and 19 Ureaplasma serovars 2, 5, 8, 9 clinical strains. File S6. Partial sequence alignment of ParC protein of 26 Ureaplasma isolates resistant to fluoroquinolones (ParC_consensus correspond to ParC protein of UPA3 and UUR8 reference strains). File S7. Partial sequence alignment of ParE protein of 26 Ureaplasma isolates resistant to fluoroquinolones (ParE_consensus correspond to ParE protein of UPA3 and UUR8 reference strains). File S8. Partial sequence alignment of L22 protein of 26 Ureaplasma isolates resistant to macrolides (L22_consensus correspond to L22 protein of UPA3 and UUR8 reference strains). (538.5 KB).

\section{Abbreviations}

AZM: Azithromycin; CIP: Ciprofloxacin; DOX: Doxycycline; ERY: Erythromycin; JOS: Josamycin; LVX: Levofloxacin; MDR: Multidrug resistance; MIC: Minimum inhibitory concentration; MXF: Moxifloxacin; OFX: Ofloxacin; QRDR: Quinolone resistance determining regions; TET: Tetracycline; UPA: Ureaplasma parvum; UUR: Ureaplasma urealyticum

\section{Acknowledgements}

This study was funded by the Tunisian Ministry of Higher Education and Scientific Research. We strongly thank the members of technical platform of Institut Pasteur de Tunis for technical assistance with automated sequencing.

\section{Authors' contributions}

$\mathrm{SB}$ and BBAM conceived and designed the experiments. BM and ABA were involved in initial isolation and identification of the isolates. S.B. performed the experiments. SB and BBAM analyzed the results and wrote the manuscript. HM contributed to the writing of the manuscript. All authors have read and approved the final manuscript.

\section{Funding}

This study was funded by the Tunisian Ministry of Higher Education and Scientific Research (LR16IPT01).

\section{Availability of data and materials}

All data generated or analysed during this study are included in this published article and its additional files.

\section{Ethics approval and consent to participate}

Not applicable.

\section{Consent for publication}

Not applicable.

\section{Competing interests}

The authors declare that they have no competing interests.

\section{Author details}

'Group of Mycoplasmas, Laboratory of Molecular Microbiology, Vaccinology, and Biotechnology Development, Institut Pasteur de Tunis, Université de Tunis El Manar, 13, Place Pasteur-B.P 74, 1002 Tunis-Belvédère, Tunisia. ${ }^{2} U n i t$ of Typing \& Genetics of Mycobacteria, Laboratory of Molecular Microbiology, Vaccinology, and Biotechnology Development, Institut Pasteur de Tunis, Université de Tunis El Manar, 13, Place Pasteur-B.P 74, 1002 Tunis-Belvédère, Tunisia.

Received: 8 May 2019 Accepted: 15 January 2020

Published online: 23 January 2020

\section{References}

1. Clegg A, Passey M, Yoannes M, Michael A. High rates of genital mycoplasma infection in the highlands of Papua New Guinea determined both by culture and by a commercial detection kit. J Clin Microbiol. 1997;35:197-200.

2. Paralanov V, Lu J, Duffy LB, Crabb DM, Shrivastava S, Methé BA, et al. Comparative genome analysis of 19 Ureaplasma urealyticum and Ureaplasma parvum strains. BMC Microbiol. 2012;12:88.

3. Waites KB, Katz B, Schelonka RL. Mycoplasmas and ureaplasmas as neonatal pathogens. Clin Microbiol Rev. 2005;18:757-89.

4. Murtha AP, Edwards JM. The role of mycoplasma and Ureaplasma in adverse pregnancy outcomes. Obstet Gynecol Clin N Am. 2014;41:615-27.

5. Waites KB, Schelonka RL, Xiao L, Grigsby PL, Novy MJ. Congenital and opportunistic infections: Ureaplasma species and mycoplasma hominis. Semin Fetal Neonatal Med. 2009;14:190-9.

6. Zhu X, Li M, Cao H, Yang X, Zhang C. Epidemiology of Ureaplasma urealyticum and mycoplasma hominis in the semen of male outpatients with reproductive disorders. Exp Ther Med. 2016;12:1165-70.

7. Mardassi BBA, Aissani N, Moalla I, Dhahri D, Dridi A, Mlik B. Evidence for the predominance of a single tet $(\mathrm{M})$ gene sequence type in tetracyclineresistant Ureaplasma parvum and mycoplasma hominis isolates from Tunisian patients. J Med Microbiol. 2012;61:1254-61.

8. Abele-Horn M, Wolff C, Dressel P, Pfaff F, Zimmermann A. Association of Ureaplasma urealyticum biovars with clinical outcome for neonates, obstetric patients, and gynecological patients with pelvic inflammatory disease. J Clin Microbiol. 1997;35:1199-202.

9. Deguchi T, Yoshida T, Miyazawa T, Yasuda M, Tamaki M, Ishiko H, et al. Association of Ureaplasma urealyticum (biovar 2) with nongonococcal urethritis. Sex Transm Dis. 2004;31:192-5.

10. Ondondo RO, Whittington WLH, Astete SG, Totten PA. Differential association of ureaplasma species with non-gonococcal urethritis in heterosexual men. Sex Transm Infect. 2010;86:271-5.

11. Kong F, Ma Z, James G, Gordon S, Gilbert GL. Species identification and subtyping of Ureaplasma parvum and Ureaplasma urealyticum using PCRbased assays. J Clin Microbiol. 2000:38:1175-9.

12. Zhang Y, Hua C, Li S-L. The relationship between the biovars and the antimicrobial resistance of Ureaplasma urealyticum in female patients with urogenital infections. J Clin Lab Anal. 2017;32(1):e22211.

13. Redelinghuys MJ, Ehlers MM, Dreyer AW, Lombaard HA, Kock MM. Antimicrobial susceptibility patterns of Ureaplasma species and mycoplasma hominis in pregnant women. BMC Infect Dis. 2014;14:171.

14. Song T, Ye A, Xie X, Huang J, Ruan Z, Kong Y, et al. Epidemiological investigation and antimicrobial susceptibility analysis of ureaplasma species and mycoplasma hominis in outpatients with genital manifestations. J Clin Pathol. 2014;67:817-20.

15. Magiorakos A-P, Srinivasan A, Carey RB, Carmeli Y, Falagas ME, Giske CG, et al. Multidrug-resistant, extensively drug-resistant and pandrug-resistant bacteria: an international expert proposal for interim standard definitions for acquired resistance. Clin Microbiol Infect. 2012;18:268-81.

16. Braam JF, van Dommelen L, Henquet CJM, van de Bovenkamp JHB, Kusters JG. Multidrug-resistant mycoplasma genitalium infections in Europe. Eur J Clin Microbiol Infect Dis. 2017;36:1565-7.

17. BBA M, Ayari H, Béjaoui-Khiari A, Mlik B, Moalla I, Amouna F. Genetic variability of the P120' surface protein gene of Mycoplasma hominis isolates recovered from Tunisian patients with uro-genital and infertility disorders. BMC Infect Dis. 2007;7 [Cited 2018 Feb 28]. Available from: http:// bmcinfectdis.biomedcentral.com/articles/10.1186/1471-2334-7-142.

18. Kong F, Zhu X, Wang W, Zhou X, Gordon S, Gilbert GL. Comparative analysis and serovar-specific identification of multiple-banded antigen genes of Ureaplasma urealyticum biovar 1. J Clin Microbiol. 1999;37:538-43. 
19. Knox CL, Giffard P, Timms P. The phylogeny of Ureaplasma urealyticum based on the mba gene fragment. Int J Syst Bacteriol. 1998;48(Pt 4): 1323-31.

20. Hall TA. BioEdit: a user-friendly biological sequence alignment editor and analysis program for windows 95/98/NT. Nucleic Acids Symp Ser. 1999;41:95-8

21. Tamura K, Stecher G, Peterson D, Filipski A, Kumar S. MEGA6: molecular evolutionary genetics analysis version 6.0. Mol Biol Evol. 2013;30:2725-9.

22. CLSI. Methods for Antimicrobial Susceptibility Testing for Human Mycoplasmas; Approved Guideline. CLSI document M43-A. Wayne: Clinical and Laboratory Standards Institute; 2011.

23. Bebear CM, Renaudin H, Charron A, Gruson D, Lefrancois M, Bebear C. In vitro activity of trovafloxacin compared to those of five antimicrobials against mycoplasmas including mycoplasma hominis and Ureaplasma urealyticum fluoroquinolone-resistant isolates that have been genetically characterized. Antimicrob Agents Chemother. 2000;44:2557-60.

24. Pereyre $\mathrm{S}$, Métifiot M, Cazanave $\mathrm{C}$, Renaudin H, Charron A, Bébéar $\mathrm{C}$, et al. Characterisation of in vitro-selected mutants of Ureaplasma parvum resistant to macrolides and related antibiotics. Int J Antimicrob Agents. 2007;29:207-11.

25. WHO Regional Office for the Eastern Mediterranean. The Work of WHO in the Eastern Mediterranean Region: annual report of the Regional Director 2013. 2013. Available from: http://applications.emro.who.int/docs/RD_ annual_Rep_2014_15459_EN.pdf?ua=1\&ua=1.

26. Mansour W. Tunisian antibiotic resistance problems: three contexts but one health. Afr Health Sci. 2018;18:1202.

27. Valentine-King MA, Brown MB. Antibacterial resistance in Ureaplasma species and mycoplasma hominis isolates from urine cultures in college-aged females. Antimicrob Agents Chemother. 2017;61:e0110417.

28. Beeton ML, Chalker VJ, Jones LC, Maxwell NC, Spiller OB. Antibiotic resistance among clinical Ureaplasma isolates recovered from neonates in England and Wales between 2007 and 2013. Antimicrob Agents Chemother. 2016;60:52-6.

29. Bayraktar MR, Ozerol IH, Gucluer N, Celik O. Prevalence and antibiotic susceptibility of mycoplasma hominis and Ureaplasma urealyticum in pregnant women. Int J Infect Dis. 2010;14:90-5.

30. Díaz L, Cabrera LE, Fernández T, Ibáñez I, Torres Y, Obregón Y, et al. Frequency and antimicrobial sensitivity of Ureaplasma urealyticum and mycoplasma hominis in patients with vaginal discharge. MEDICC Rev. 2013; 15:45-7.

31. Dégrange $S$, Renaudin H, Charron A, Bébéar C, Bébéar CM. Tetracycline resistance in Ureaplasma spp. and mycoplasma hominis: prevalence in Bordeaux, France, from 1999 to 2002 and description of two tet (M)-positive isolates of M. hominis susceptible to tetracyclines. Antimicrob Agents Chemother. 2008;52:742-4.

32. Fernández J, Karau MJ, Cunningham SA, Greenwood-Quaintance KE, Patel R. Antimicrobial susceptibility and Clonality of clinical Ureaplasma isolates in the United States. Antimicrob Agents Chemother. 2016;60: 4793-8.

33. Song J, Qiao Y, Kong Y, Ruan Z, Huang J, Song T, et al. Frequent topoisomerase IV mutations associated with fluoroquinolone resistance in Ureaplasma species. J Med Microbiol. 2015;64:1315-20.

34. Zeng $X-Y$, Xin N, Tong $X-N$, Wang J-Y, Liu Z-W. Prevalence and antibiotic susceptibility of Ureaplasma urealyticum and mycoplasma hominis in Xi'an, China. Eur J Clin Microbiol Infect Dis. 2016;35:1941-7.

35. O'Connor S, Rifkin D, Yang YH, Wang JF, Levine OS, Dowell SF. Physician control of pediatric antimicrobial use in Beijing, China, and its rural environs. Pediatr Infect Dis J. 2001;20:679-84.

36. Piddock $L$ J. Mechanisms of fluoroquinolone resistance: an update 1994 1998. Drugs. 1999;58(Suppl 2):11-8.

37. Kawai Y, Nakura Y, Wakimoto T, Nomiyama M, Tokuda T, Takayanagi $T$, et al. In Vitro Activity of Five Quinolones and Analysis of the Quinolone Resistance-Determining Regions of gyrA, gyrB, parC, and parE in Ureaplasma parvum and Ureaplasma urealyticum Clinical Isolates from Perinatal Patients in Japan. Antimicrob Agents Chemother. 2015;59:2358-64.

38. Chang J, Yu JK, Song C, Park IY, Park Y-J. Prevalence and Antimicrobial Susceptibility of Genital Mycoplasmataceae in Korean Women: Correlation between Phenotypic Test and Resistance Genes. Ann Clin Microbiol. 2016; 19:13.

39. Damião Gouveia $A C$, Unemo $M$, Jensen JS. In vitro activity of zoliflodacin (ETX0914) against macrolide-resistant, fluoroquinoloneresistant and antimicrobial-susceptible mycoplasma genitalium strains. J Antimicrob Chemother. 2018;73:1291-4.

40. Xiao L, Crabb DM, Duffy LB, Paralanov V, Glass Jl, Hamilos DL, et al. Mutations in ribosomal proteins and ribosomal RNA confer macrolide resistance in human Ureaplasma spp. Int J Antimicrob Agents. 2011;37: $377-9$.

\section{Publisher's Note}

Springer Nature remains neutral with regard to jurisdictional claims in published maps and institutional affiliations.

\section{Ready to submit your research? Choose BMC and benefit from:}

- fast, convenient online submission

- thorough peer review by experienced researchers in your field

- rapid publication on acceptance

- support for research data, including large and complex data types

- gold Open Access which fosters wider collaboration and increased citations

- maximum visibility for your research: over $100 \mathrm{M}$ website views per year

At BMC, research is always in progress.

Learn more biomedcentral.com/submissions 